\title{
Impact of apical and mid-ventricular transmural infarcts in patients with acute myocardial infarction determined by using late gadolinium enhancement combined with feature tracking magnetic resonance
}

\author{
Junchao $\mathrm{Li}^{1}$, Menglu $\mathrm{Li}^{2}$, Yundai Chen ${ }^{1}$, Liuquan Cheng ${ }^{2}$, Geng Qian ${ }^{1}$, Jiayue $\mathrm{Li}^{1}$, Xiao Zhou ${ }^{1}$, \\ Bohan Liu ${ }^{3,4}$, Wei Dong ${ }^{1 \wedge}$
}

${ }^{1}$ Senior Department of Cardiology, the Sixth Medical Center of PLA General Hospital, Beijing, China; ${ }^{2}$ Department of Radiology, the Sixth Medical Center of PLA General Hospital, Beijing, China; ${ }^{3}$ Department of Cardiovascular Surgery, Chinese PLA General Hospital, Beijing, China; ${ }^{4}$ Beijing Key Laboratory of Chronic Heart Failure Precision Medicine, Chinese PLA General Hospital, Beijing, China

Contributions: (I) Conception and design: Junchao Li, W Dong, Y Chen; (II) Administrative support: W Dong; (III) Provision of study materials or patients: Junchao Li, W Dong, Y Chen; (IV) Collection and assembly of data: W Dong, L Cheng, G Qian, Junchao Li, M Li, Jiayue Li, B Liu; (V) Data analysis and interpretation: W Dong, Y Chen, X Zhou, L Cheng; (VI) Manuscript writing: All authors; (VII) Final approval of manuscript: All authors.

Correspondence to: Wei Dong. Senior Department of Cardiology, the Sixth Medical Center PLA General Hospital, No. 6, Fucheng Road, Haidian District, Beijing 100048, China. Email: 301dongw@sina.com.

Background: To analyze the relationship between left ventricular (LV) myocardial strain and transmurality
of myocardial infarction at three circular sections (basal, mid-ventricular, apical) by a combined analysis
of cardiac magnetic resonance feature tracking (CMR-FT) and late gadolinium enhancement (LGE)
information in a cohort of ST-elevation acute myocardial infarction (STEMI) patients after primary
percutaneous coronary intervention (PPCI).
Methods: In all, 136 patients with STEMI who underwent PPCI within 12 hours of symptom onset were
included. CMR-FT and LGE-MRI were performed $5 \pm 2$ days after PCI for measuring regional and global
myocardial strain indexes and transmural extent. Multivariate regression analysis and Kaplan-Meier survival
analysis were performed.

Results: Regional radial and circumferential strain decreased with increasing transmurality of myocardial infarction irrespective of basal, mid-ventricular, or apical segments. Segmental longitudinal strain was significantly decreased in the transmural infarcted segments only at the apical and mid-ventricular levels. A significant correlation was found between the number of transmural infarcts and global strain parameters in the apical and mid-ventricular portions. Transmural infarcted segments in apical + mid-ventricular portions $>2$ was related to an increased risk of cardiac events in patients with STEMI following PPCI than those $\leq 2$. GLS was found to be an independent predictor of cardiac events in these patients.

Conclusions: The number of transmural infarcted segments in apical + mid-ventricular portions affects LV global function and prognosis. Global longitudinal strain (GLS) is a significant predictor of adverse events after PPCI for STEMI. Morphologic and functional data fused to study complex pathophysiologic processes of LV early after STEMI may help in risk stratification of patients.

Keywords: Feature tracking technique; cardiac magnetic resonance (CMR); acute myocardial infarction (AMI); myocardial strain

\footnotetext{
$\wedge$ ORCID: 0000-0003-4525-1105.
} 
Submitted Jun 20, 2021. Accepted for publication Nov 03, 2021.

doi: $10.21037 / \mathrm{cdt}-21-382$

View this article at: https://dx.doi.org/10.21037/cdt-21-382

\section{Introduction}

ST-elevation acute myocardial infarction (STEMI) is a type of acute myocardial infarction (AMI) with symptoms characteristic of myocardial ischemia along with persistent electrocardiographic ST elevation (1). Left ventricular (LV) dysfunction remains common and associated with a worse prognosis even the current large-scale use of primary percutaneous coronary intervention (PPCI) for patients with STEMI (2). Myocardial strain is a measure of myocardial deformation and can represent cardiac function (3). LV global longitudinal strain (GLS) is more sensitive and reproducible for assessment of $L V$ function than $L V$ ejection fraction (LVEF) (4). Recognition of degrees of infarct transmurality allows the definition of myocardial viability, which is associated with functional recovery and prognosis after revascularization (5). A comprehensive evaluation of myocardial function and viability is clinically important in the management and prognosis of patients.

Cardiovascular magnetic resonance (CMR) has emerged as the imaging modality of choice to study different stages of MI (6). Late gadolinium enhancement (LGE)-CMR is the current gold standard for differentiating reversible and irreversible myocardial injury $(7,8)$. The emerging CMR feature tracking imaging (CMR-FT) is a novel tissue tracking technique that allows precise quantification of global and regional myocardial strain using routine cine balanced steady-state free precession (bSSFP) techniques (9). A recent study by Zou et al. suggested that myocardial strain derived from CMR tissue-tracking can differentiate between subendocardial and transmural infarction (7). Certain researches have indicated that regional and global strain correlates with the size and transmural extent of myocardial infarction (10-12). However, there is a paucity of data regarding the relationship between myocardial strain and various degrees of myocardial infarct transmurality in the apical, mid-ventricular, or basal segments. We hypothesized that infarct severity at different segmental levels could have different effects on the myocardial function, which in turn affects prognosis.

In this study, we performed a combined analysis of CMR-FT and LGE information to evaluate the relationship between regional or global myocardial strains and the transmural extent of myocardial infarction at three circular sections (basal, mid-ventricular, apical), and also to comprehensively evaluate their predictive effect on prognosis in patients with STEMI after PPCI.

We present the following article in accordance with the STROBE reporting checklist (available at https://cdt. amegroups.com/article/view/10.21037/cdt-21-382/rc).

\section{Methods}

\section{Study population}

From February 2014 to April 2019, 136 patients with STEMI who underwent PPCI within 12 hours of symptom onset were included. STEMI is diagnosed based on the presence of chest pain $>30$ minutes, ST-segment elevation of $0.1 \mathrm{mV}$ or more in at least two contiguous limb leads, or ST-segment elevation of $\geq 0.2 \mathrm{mV}$ in at least two contiguous chest leads on electrocardiogram, and elevated serum troponin $\mathrm{T}$ levels. The study inclusion criteria were as follows: age 18 years or older; chest pain lasting for $<12$ hours; diagnosed with acute myocardial infarction; PPCI performed within 12 hours after symptom onset; CMR cine sequence and late gadolinium enhancement MRI (LGE-MRI) performed 5 \pm 2 days after STEMI; and provision of signed informed consent. Patients with a history of previous myocardial infarction, complete bundle branch block, permanent atrial fibrillation, pacemaker implantation, severe valvular heart disease, and congenital heart disease were excluded.

The study was conducted in accordance with the Declaration of Helsinki (as revised in 2013). This study obtained approval from the Medical Ethics Committee of Chinese PLA General Hospital (No. S2017-127-01), and all patients provided written informed consent before participation in this investigation.

\section{Primary PCI}

PPCI was performed according to the current PCI practice guideline. All patients received a loading dose of $300 \mathrm{mg}$ aspirin and $600 \mathrm{mg}$ clopidogrel before PCI. Drug-eluting stent implantation was preferred as the first choice. 

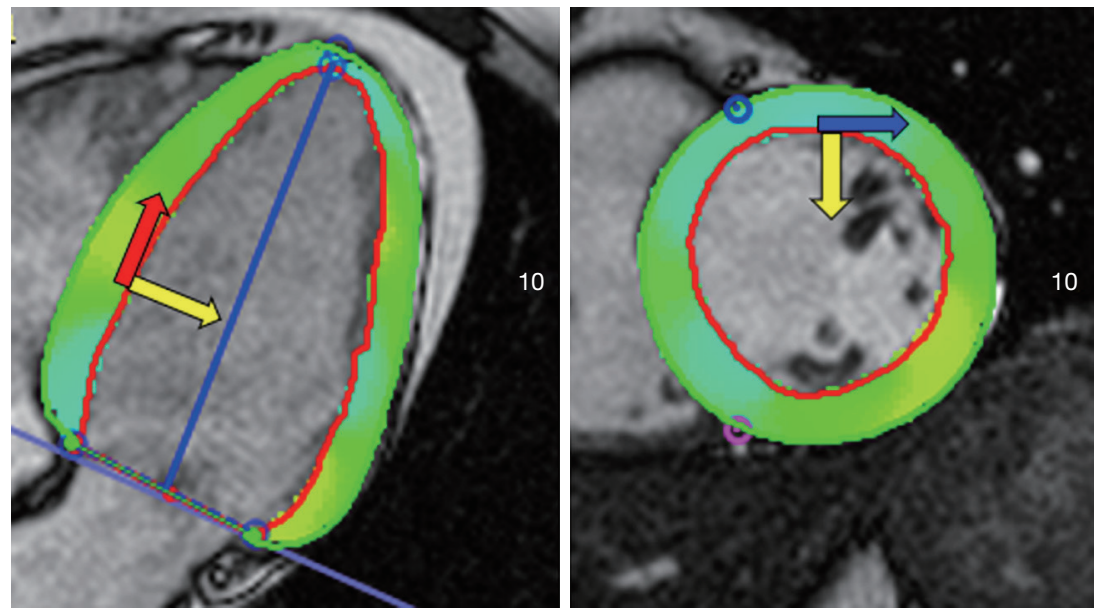

Figure 1 CVI42 for analysing the long and short axis cine sequences. The red curve represents the endocardium, yellow arrow represents radial strain, red arrow represents longitudinal strain, and the blue arrow represents circumferential strain.

\section{CMR image acquisition}

CMR imaging was performed using a 1.5 -T scanner (SIGNA EXCITE HD, GE Medical Systems, Milwaukee, WI, USA). In each subject, a bSSFP cine sequence was used to acquire a stack of slices in long axis 2-, 3- and 4-chamber views (13) and in a ventricular short-axis plane to obtain whole coverage of basal segment of LV (from the area extending from the mitral annulus to the tips of papillary muscles at end-diastole), middle segment of LV (the region that includes the entire length of the papillary muscles at end-diastole) and apical segment of LV (beyond the papillary muscles but before the cavity ends at end-diastole), according to a modified 16-segment LV model (omitting the apical cap) based on the standard 17-segment model defined by the American Heart Association (14,15). The cine imaging was performed with end-expiratory breathholding for 15-18 s. The acquisition parameters were as follows: the minimum repetition time (TR) of $3.7 \mathrm{~ms}$, the minimum echo time (TE) of $1.87 \mathrm{~ms}$, flip angle of $60^{\circ}$, field of view (FOV) of $32 \mathrm{~cm} \times 28 \mathrm{~cm}$, matrix of $228 \times 194$, and slice thickness of $8 \mathrm{~mm}$. These measurements were obtained for 24 evenly spaced cardiac phase of the R-R interval (i.e., one heart-beat).

For the LGE studies, gadobenate dimeglumine (GdBOPTA) was injected intravenously as a rapid bolus at a dose of $0.2 \mathrm{mmol} / \mathrm{kg}$ body weight using a CMR highpressure injector at an injection rate of $3.5 \mathrm{~mL} / \mathrm{s}$. At $10 \mathrm{~min}$ post-contrast administration, delayed enhanced imaging scans were performed with breath-holding and the LGE images in a short axis stack covering the entire $L V$ were generated with the following parameters: $T R=2 R-R$ intervals; flip angle, $20^{\circ}$; FOV, $36 \mathrm{~cm} \times 27 \mathrm{~cm}$; matrix, $256 \times 192$; inversion time, $200-360 \mathrm{~ms}$. Slice thickness was $8 \mathrm{~mm}$ with a $2 \mathrm{~mm}$ gap.

\section{CMR image analysis}

CMR feature tracking strain analysis was performed using dedicated tissue tracking software (CVI42, version 5.12.1, Circle Cardiovascular Imaging Inc., Canada) (Figure 1). LV endocardial and epicardial contours were manually traced at the end-diastole followed by automatic propagating contours to the whole cardiac cycle. Contours were visually reviewed and manually corrected by border adjustment when automatic tracking failed. Regional cardiac function indicators, including circumferential, radial, and longitudinal strain peak values for basal, mid-ventricular, and apical segments were obtained. An average value for all segments was reported as a global strain value for the entire LV. Intra-observer variability and interobserver reproducibility of global and segmental strain analysis were assessed by two observers on a subset of 22 randomly selected cases. To assess the intra-observer variability, one observer blinded to the initial study result repeated the analysis after two months.

CVI42 software (version 5.12.1, Circle Cardiovascular Imaging Inc., Canada) was also used to analyze the LGE images. The LV endocardial and epicardial borders were traced manually, and the infarct area was detected 
automatically. LGE quantification was based on the full-width at half-maximum (FWHM) technique (16). The transmural extent of infarction of each myocardial segment and the size of the infarction area accounting for the percentage of LV myocardium were recorded. The segmental transmurality of myocardial infarction was determined as a relative amount of contrast-enhanced myocardial tissue ( $0 \%$ : no infarction; $1-50 \%$ : nontransmural infarction; 51-100\%: transmural infarction) (Figure S1).

\section{Follow-up}

The adverse cardiovascular events of 136 patients were collected by remote follow-up. Information on the outcome was obtained and confirmed using medical record files uploaded by the patients or their family members. The events recorded in the medical records were supervised by healthcare personnel.

\section{Statistical analysis}

Data analysis was completed using SPSS Statistics v.24.0 software (IBM Corp., Armonk, NY, USA). ShapiroWilk test was applied to assess data normality. Results are expressed as mean \pm standard deviation (SD) or median (interquartile range, IQR). Normally distributed continuous variables between two groups were compared using the student's $t$-test and Wilcoxon rank-sum test was used to compare continuous variables of non-normally distributed as appropriate. Statistical differences between multiple groups were tested with one-way analysis of variance (ANOVA) or the Kruskal-Wallis test, followed by a post hoc test with Bonferroni correction. Categorical variables were expressed as numbers with percentages in parentheses and analyzed with the Chi-square test or Fischer's exact test as appropriate. To evaluate inter-observer and intra-observer consistency and reliability, Cronbach's alpha coefficient and intraclass correlation coefficient (ICC) are used, respectively. Internal consistency was defined as inadmissible (Cronbach's alpha coefficient $<0.6)$, weak $(0.6-0.7)$, reasonable $(0.7-0.8)$, good (0.8-0.9), or excellent (Cronbach's alpha coefficient $>0.9)$ (17). The value of ICC $\geq 0.75$ was defined as excellent (ICC), 0.6-0.74 was good, 0.4-0.59 was fair, and ICC $<0.40$ was poor (18). A Spearman correlation analysis was used to determine the correlation between global strain parameters and the number of myocardial infarction segments in the basal, mid-ventricular, or apical portions of the LV. The correlation was interpreted as negligible $(\mathrm{r}<0.10)$, weak $(0.10-0.39)$, moderate $(0.40-0.69)$, strong (0.70-0.89), or very strong ( $r \geq 0.90)$ (19). A stepwise multivariate Cox proportional hazards model was used to investigate prognostic predictors. The Kaplan-Meier curve was constructed to depict the impact of the number of transmural infarcted segments on clinical cardiac outcomes, followed by a log-rank test for comparison. A $\mathrm{P}$ value of less than 0.05 was considered statistically significant.

\section{Results}

\section{Patient characteristics}

Demographics of the 136 patients are displayed in Table 1 . The mean age of the study population was $55.9 \pm 11.6$ years. Almost $90 \%$ of patients in the population were male. Culprit lesions in the left anterior descending, right coronary artery, and left circumflex artery were found in 63, 57, and 16 cases, respectively. By CMR scans, 775 non-infarcted segments, 1,053 non-transmural infarcted segments, and 348 transmural infarcted segments were detected.

\section{Inter-observer and intra-observer consistency and reliability analysis}

As shown in Table 2, longitudinal strain, radial strain, and circumferential strain analysis showed good or excellent intraobserver and interobserver agreement (ICC >0.74). On the other hand, longitudinal strain, radial strain, and circumferential strain analysis showed good or excellent internal consistency (Cronbach's alpha coefficient $>0.8$ ).

\section{Relationship between regional myocardial strain and transmurality of myocardial infarction}

We compared circumferential strain, longitudinal strain, and radial strain values, respectively, based on different transmurality states of infarcted tissue for the basal, midventricular, and apical segments. Results are summarized in Table 3. A representative bull eye plot with strain curves showing strain calculation in non-transmural and transmural infarcts is shown in Figure 2.

Radial strain varied in amplitude with highest values in the non-infarcted segments and lowest in segments with transmural infarction at the apical, mid-ventricular and basal levels [apical: $35.46 \%$ (24.85\%) vs. $28.48 \%$ (33.54\%) vs. 
Table 1 Basic characteristics of 136 patients with STEMI

\begin{tabular}{|c|c|}
\hline Characteristics & Total, $n=136$ \\
\hline Age (years) & $55.9 \pm 11.6$ \\
\hline Sex (male/female) & $123 / 13$ \\
\hline BMI $\left(\mathrm{kg} / \mathrm{m}^{2}\right)$ & $26.95 \pm 3.68$ \\
\hline Heart rate (beats per minute) & $71.22 \pm 11.38$ \\
\hline \multicolumn{2}{|l|}{ Past history } \\
\hline Diabetes & 26 \\
\hline Hypertension & 51 \\
\hline Hyperlipidemia & 38 \\
\hline Cerebral infarction & 4 \\
\hline \multicolumn{2}{|l|}{ Culprit vessels } \\
\hline LAD & 63 \\
\hline $\mathrm{RCA}$ & 57 \\
\hline LCx & 16 \\
\hline \multicolumn{2}{|l|}{ Baseline CMR } \\
\hline $\mathrm{EF}(\%)$ & $48.96 \pm 9.60$ \\
\hline Infarct size (\%), median (IQR) & $13.00(15.72)$ \\
\hline Non-infarcted segments & 775 \\
\hline Non-transmural infarcted segments & 1,053 \\
\hline Transmural infarcted segments & 348 \\
\hline
\end{tabular}

Continuous variables with normal distribution were presented as mean \pm SD (standard deviation); non-normal variables were reported as median [interquartile range (IQR)]. STEMI, STelevation acute myocardial infarction; BMI, body mass index; LAD, left anterior descending; RCA, right coronary artery; LCX, left circumflex artery; CMR, cardiovascular magnetic resonance; $\mathrm{EF}$, ejection fraction.

Table 2 Intra-observer and inter-observer variability of strain analysis

\begin{tabular}{lccccc}
\hline \multirow{2}{*}{ Variable } & \multicolumn{2}{c}{ Inter-observer } & & \multicolumn{2}{c}{ Intra-observer } \\
\cline { 2 - 3 } \cline { 5 - 6 } & ICC & $\begin{array}{c}\text { Cronbach's } \\
\text { alpha }\end{array}$ & & ICC & $\begin{array}{c}\text { Cronbach's } \\
\text { alpha }\end{array}$ \\
\hline Radial strain & 0.899 & 0.944 & 0.912 & 0.954 \\
$\begin{array}{l}\text { Circumferential } \\
\text { strain }\end{array}$ & 0.854 & 0.921 & & 0.910 & 0.950 \\
Longitudinal strain & 0.742 & 0.824 & 0.761 & 0.813 \\
\hline
\end{tabular}

ICC, intraclass correlation coefficient.

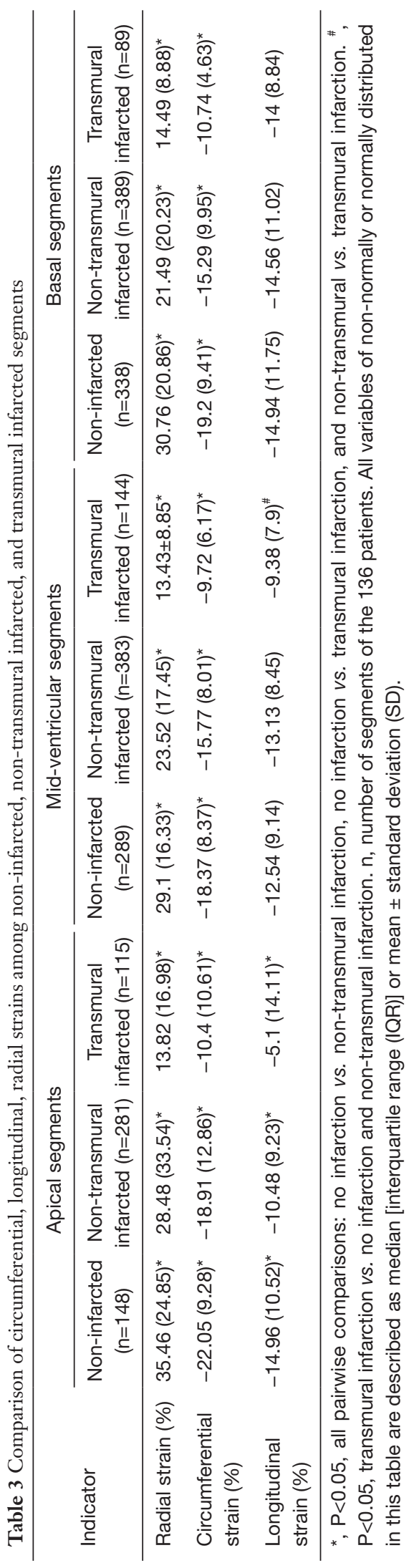



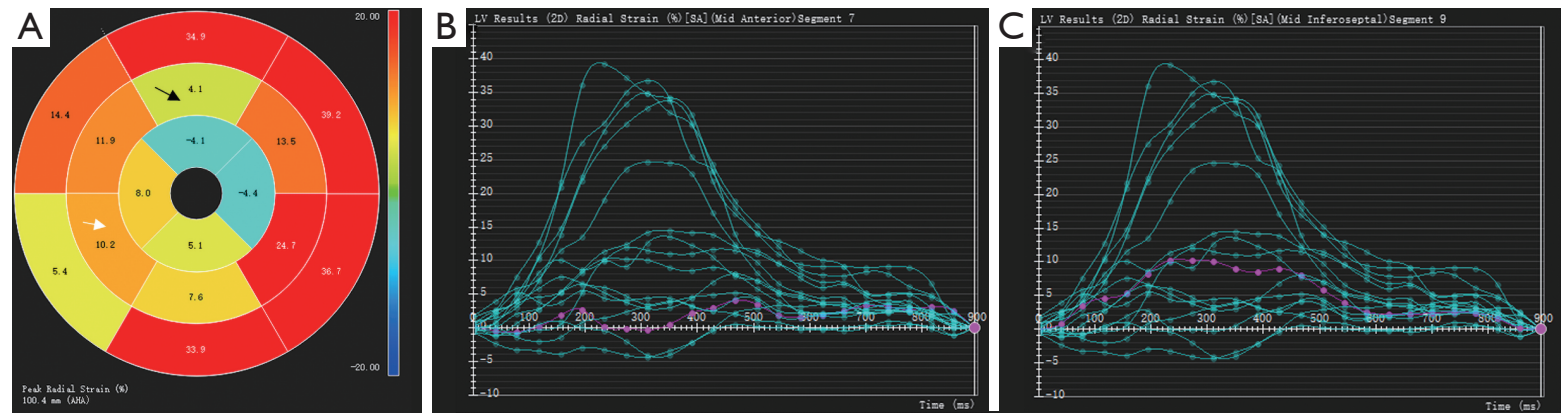

Figure 2 A representative bull eye plot with strain curves showing strain calculation in non-transmural and transmural infarcts. (A) A bull eye plot showing segmental peak systolic radial strain. The black arrow indicates AHA segment 7 (a transmural infarcted segment with the transmural extent of 93.5\%). The white arrow indicates AHA segment 9 (a non-transmural infarcted segment with the transmural extent of $44.0 \%$ ). (B) Peak radial strain curve (purple curve), corresponding to the AHA segment 7. (C) Peak radial strain curve (purple curve), corresponding to the AHA segment 9.

13.82\% (16.98\%), $\mathrm{P}<0.05$; mid-ventricular: $29.1 \%$ (16.33\%) vs. $23.52 \%(17.45 \%)$ vs. $13.43 \% \pm 8.85 \%, \mathrm{P}<0.05$; basal: $30.76 \%$ (20.86\%) vs. $21.49 \%$ (20.23\%) vs. $14.49 \%$ (8.88\%), $\mathrm{P}<0.05]$. A similar distribution pattern was present for the circumferential strain with the highest value (in absolute values) in the non-infarcted segments and lowest in the transmural infarcted segments at the basal, mid-ventricular, and apical levels $(\mathrm{P}<0.05)$. At the apical level, longitudinal strain (in absolute values) had a similar trend of decline from non-infarcted segments to transmural infarcted segments $(\mathrm{P}<0.05)$. At the mid-ventricular level, longitudinal strain values in segments without infarction and segments with non-transmural infarction were higher than transmural infarction segments $(\mathrm{P}<0.05)$; however, longitudinal strain did not differentiate between non-infarcted segments and non-transmural infarcted segments $(\mathrm{P}>0.05)$. Moreover, the longitudinal strain was not different between non-infarcted segments, segments with non-transmural infarction, and those with transmural infarction at the basal level $[-14.94 \%$ (11.75\%), $-14.56 \%$ (11.02\%), $-14 \%$ (8.84\%), respectively, $\mathrm{P}>0.05]$.

\section{Correlation of global myocardial strain with myocardial infarction segments}

As shown in Table 4, we found GLS showing moderate positive correlation to number of transmural infarcted segments in the apical and mid-ventricular portions $(\mathrm{P}<0.0001, \mathrm{r}=0.482 ; \mathrm{P}<0.0001, \mathrm{r}=0.613)$. GCS showed a positive correlation with the number of transmural infarcts in the apical and mid-ventricular portions $(\mathrm{P}<0.0001$, $\mathrm{r}=0.531 ; \mathrm{P}<0.0001, \mathrm{r}=0.555)$. That is, GLS and GCS in absolute values decreased with an increased number of transmural infarcts in the apical and mid-ventricular portions. An inverse relationship was found between GRS and transmural infarcts in the apical or mid-ventricular portion $(\mathrm{P}<0.0001, \mathrm{r}=-0.561 ; \mathrm{P}<0.0001, \mathrm{r}=-0.515)$. Only a weak correlation was found between non-transmural or transmural infarcted segments and global strain parameters (GRS and GCS) in the basal section of the LV.

\section{Correlation of $L V$ index and cardiac event occurrence}

Of the 136 patients, 21 (15.4\%) were lost to follow-up. The average follow-up period of the remaining 115 patients was $2.24 \pm 1.17$ years. Of these, 32 patients experienced adverse cardiovascular events (cardiac death, 3; heart failure, 2; recurrent myocardial infarction, 1; hospitalization due to recurrent ischemic events, 26).

By a stepwise multivariate Cox regression analysis (Table 5), GLS (HR =1.211, 95\% CI: 1.015-1.444, P=0.033), $\mathrm{EF}(\mathrm{HR}=0.002,95 \% \mathrm{CI}: 0.000-0.388, \mathrm{P}=0.020)$ and age (HR $=1.044$, 95\% CI: $1.005-1.086, \mathrm{P}=0.028$ ) were found to be independent predictors of cardiac events in patients with STEMI after PPCI. Furthermore, we investigated the effect of a specified number of apical and mid-ventricular transmural infarcts on affecting prognosis. The number of transmural infarcted segments in apical + mid-ventricular portions $>2$ was related to an increased risk of cardiac events in patients with STEMI following PPCI than those with transmural infarcted segments in apical + mid-ventricular portions $\leq 2$ (Figure 3, $\mathrm{P}=0.03$ by log-rank test). 
Table 4 The correlation of global strain parameters and number of myocardial infarction segments at three circular sections (basal, midventricular, apical)

\begin{tabular}{|c|c|c|c|c|c|c|}
\hline Number of segments & \multicolumn{2}{|c|}{ GRS } & \multicolumn{2}{|c|}{ GCS } & \multicolumn{2}{|c|}{ GLS } \\
\hline Non-transmural infarcted segments in the apical portion & 0.107 & 0.215 & -0.120 & 0.165 & -0.099 & 0.250 \\
\hline Transmural infarcted segments in the apical portion & -0.561 & $<0.0001$ & 0.531 & $<0.0001$ & 0.482 & $<0.0001$ \\
\hline Non-transmural infarcted segments in the mid-ventricular portion & -0.054 & 0.531 & 0.108 & 0.211 & -0.053 & 0.539 \\
\hline Non-transmural infarcted segments in the basal portion & -0.197 & 0.022 & 0.289 & 0.001 & 0.082 & 0.345 \\
\hline Transmural infarcted segments in the basal portion & -0.087 & 0.313 & 0.227 & 0.008 & 0.168 & 0.051 \\
\hline
\end{tabular}

GRS, global radial strain; GCS, global circumferential strain; GLS, global longitudinal strain.

Table 5 Univariate and multivariate Cox proportional hazard regression analysis

\begin{tabular}{|c|c|c|c|c|c|c|}
\hline Variable & \multicolumn{3}{|c|}{ Univariate analysis } & \multicolumn{3}{|c|}{ Multivariate analysis } \\
\hline Age & 1.036 & $1.003-1.069$ & 0.033 & 1.044 & $1.005-1.086$ & 0.028 \\
\hline BMI & 1.039 & $0.935-1.155$ & 0.478 & & & \\
\hline \multicolumn{7}{|l|}{ Past history } \\
\hline Hypertension & 0.966 & $0.397-2.350$ & 0.940 & & & \\
\hline Hyperlipidemia & 0.939 & $0.421-2.093$ & 0.877 & & & \\
\hline Cerebral infarction & 2.432 & $0.577-10.256$ & 0.226 & & & \\
\hline \multicolumn{7}{|l|}{ Culprit vessels } \\
\hline LCx & 0.729 & $0.222-2.396$ & 0.603 & & & \\
\hline \multicolumn{7}{|l|}{ CMR parameters } \\
\hline $\mathrm{EF}$ & 0.001 & $0.000-0.029$ & $<0.001$ & 0.002 & $0.000-0.388$ & 0.020 \\
\hline GRS & 0.941 & $0.888-0.997$ & 0.041 & 1.073 & $0.948-1.214$ & 0.264 \\
\hline GCS & 1.122 & $1.009-1.247$ & 0.034 & 1.073 & $0.873-1.318$ & 0.504 \\
\hline GLS & 1.270 & $1.113-1.450$ & $<0.001$ & 1.211 & $1.015-1.444$ & 0.033 \\
\hline
\end{tabular}

BMI, body mass index; LAD, left anterior descending; RCA, right coronary artery; LCx, left circumflex artery; CMR, cardiovascular magnetic resonance; EF, ejection fraction; GRS, global radial strain; GCS, global circumferential strain; GLS, global longitudinal strain.

\section{Discussion}

The present study shows that regional myocardial strain in patients with STEMI after PPCI differs among noninfarcted, non-transmural, and transmural infarction in the apical, mid-ventricular, and basal portions of the LV. An increased number of transmural infarcts in the apical and mid-ventricular portions was significantly associated with the extent or severity of global LV dysfunction. Patients with transmural infarcted segments in apical + midventricular portions $>2$ were at an elevated risk of cardiac 


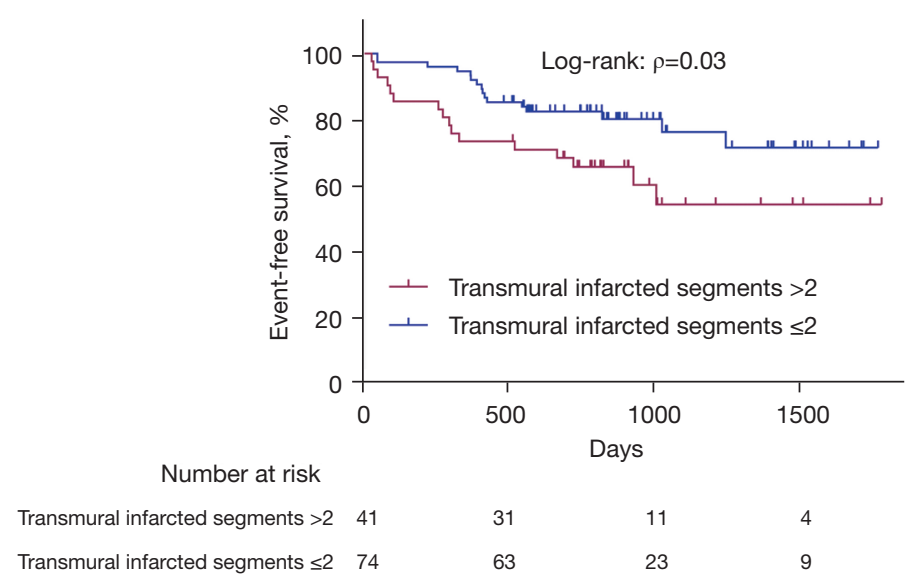

Figure 3 Kaplan-Meier curves for incidence of cardiac events for patients stratified by the number of transmural infarcted segments in apical + mid-ventricular portions $>2$ versus $\leq 2$.

events than those with transmural infarcted segments in apical + mid-ventricular portions $\leq 2$. In addition, GLS was an independent prognosticator of adverse clinical events of patients with STEMI following PPCI.

CMR has the unique and powerful ability to evaluate the size and transmural extent of infarction with the LGE technique (20), revealing the histological characteristics of myocardial injury. CMR-FT is a non-invasive approach that allows quantitative strain analysis of myocardial function (21). In this study, CMR-FT in conjunction with LGE was used to simultaneously evaluate morphologic and functional data to study complex pathophysiologic processes of LV early after STEMI. We found the peak values of radial strain and circumferential strain (in absolute values) with the highest value in the non-infarcted segments and lowest in the transmural infarcted segments at the basal, mid-ventricular, and apical sections; regional longitudinal strain presented different changes at three circular parts. Sjøli et al. (22) used Doppler and speckle tracking to measure strain and used CMR to measure infarct transmurality in 36 patients with STEMI treated with thrombolysis. The results indicated that segmental circumferential and longitudinal strains were decreased with increased levels of infarct transmurality. The change in circumferential strain in our study was similar to previous studies (22-24) which demonstrate that circumferential strain reflects the degree of transmurality. In addition, our results showed that regional radial strain was noticeably reduced with an increased degree of transmurality across three different circular parts of LV. However, we found that segmental longitudinal strain was significantly decreased in the transmural infarcted segments only at the apical and mid-ventricular levels. We may reasonably assume that longitudinal strain was less affected by infarct transmurality in the basal segment.

Some studies have shown that the long axis strain assessed by MRI provides an important supplement for the prediction of cardiac events and risk stratification in nonischemic cardiomyopathy (25). Our study observed that GLS assessed by CMR-FT independently predicted the incidence of adverse events. This is consistent with a recent study demonstrating the significance of GLS in predicting postoperative outcomes (26). Similarly, a recent study by Reindl et al. demonstrated that GLS detected by CMRFT strongly and independently predicted clinical outcomes following STEMI patients (27). Future studies are necessary to assess the prognostic implications of these findings and the ideal cut-off value for GLS.

Interestingly, the obtained results revealed moderate inverse relationships between GRS, GCS, or GLS in absolute values and the number of transmural infarcts in the apical and mid-ventricular portions. The results indicated that infarct severity in the apical and midventricular sections significantly affected cardiac function. AMI occurs with abnormal myocardial systolic contraction, including hypokinesis, cessation of myocardial contraction in affected regions, or paradoxical motion (28). After PPCI, reperfusion of the infarct area can repair the infarcted region, reduce the infarct expansion, and bring about a certain degree of recovery in contraction. The blood flow of the apical segment is mainly supplied by the distal part of the coronary artery. After patency of the vessels, the 
blood supply recovery of the infarcted tissue in the apical segment is inferior to that of the basal segment. Therefore, the repair of the infarcted tissue in the apical segment is inferior to that of the infarcted tissue in the basal segment. Myocardial no-reflow may be more likely to occur in the distal segment (29). Moreover, the apex of the heart is the thinnest part of the ventricle (30), and it is also the most vulnerable area to be damaged by infarct expansion. After PPCI, the recovery of the local function of the apical infarcted area is worse than that of the basal segment, and the local function of the apical infarcted segment is affected, which affects the overall cardiac function indexes. Evidence indicates that wall motion abnormalities most involved the mid-ventricular segment (31). In our study, we found that transmural infarcted segments $>2$ at the middle-to-apical portions were associated with an increased risk of cardiac events in patients with STEMI following PPCI. This may add important information to the risk stratification in these patients.

This study has a few limitations. First, this was a single-center study with a small sample size. Second, only Circle CVI42 was used to measure the myocardial strain. The results can be validated by other software due to substantial variability among vendors (32). However, there were good to excellent inter-observer and intra-observer agreements for CMR-FT-derived measurements in this study population, which supports the reliability of the findings. Third, this study did not include CMR evaluation during follow-up. Future studies are needed to investigate myocardial changes by comprehensive follow-up CMR examinations.

In conclusion, this study demonstrated the correlation of regional myocardial strain and transmural extent of myocardial infarction both detected by CMR in STEMI patients following PPCI. Transmural infarcted segments $>2$ in apical plus mid-ventricular portions may affect $\mathrm{LV}$ global function and prognosis of patients. GLS provides independent prognostic value for patients with STEMI. By a combined analysis of LGE and CMR-FT information, morphologic and functional imaging can be fused, which may more accurately predict viable segments and clinical outcomes.

\section{Acknowledgments}

Funding: This work was supported by National Key R\&D Program of China (No. 2018YFC0910700).

\section{Footnote}

Reporting Checklist: The authors have completed the STROBE reporting checklist. Available at https://cdt. amegroups.com/article/view/10.21037/cdt-21-382/rc

Data Sharing Statement: Available at https://cdt.amegroups. com/article/view/10.21037/cdt-21-382/dss

Peer Review File: Available at https://cdt.amegroups.com/ article/view/10.21037/cdt-21-382/prf

Conflicts of Interest: All authors have completed the ICMJE uniform disclosure form (available at https://cdt.amegroups. com/article/view/10.21037/cdt-21-382/coif). The authors have no conflicts of interest to declare.

Ethical Statement: The authors are accountable for all aspects of the work in ensuring that questions related to the accuracy or integrity of any part of the work are appropriately investigated and resolved. The study was conducted in accordance with the Declaration of Helsinki (as revised in 2013). The study was approved by the Medical Ethics Committee of Chinese PLA General Hospital (No. S2017-127-01) and informed consent was taken from all individual participants before participation in this investigation.

Open Access Statement: This is an Open Access article distributed in accordance with the Creative Commons Attribution-NonCommercial-NoDerivs 4.0 International License (CC BY-NC-ND 4.0), which permits the noncommercial replication and distribution of the article with the strict proviso that no changes or edits are made and the original work is properly cited (including links to both the formal publication through the relevant DOI and the license). See: https://creativecommons.org/licenses/by-nc-nd/4.0/.

\section{References}

1. Endorsed by the Latin American Society of Interventional Cardiology; PCI WRITING COMMITTEE; Levine GN, et al. 2015 ACC/AHA/SCAI focused update on primary percutaneous coronary intervention for patients with ST-elevation myocardial Infarction: An update of the 2011 ACCF/AHA/SCAI guideline for percutaneous coronary intervention and the 2013 ACCF/AHA guideline 
for the management of ST-elevation myocardial infarction: A report of the American College of Cardiology/ American Heart Association Task Force on Clinical Practice Guidelines and the Society for Cardiovascular Angiography and Interventions. Catheter Cardiovasc Interv 2016;87:1001-19.

2. Kim DH, Park CB, Jin ES, et al. Predictors of decreased left ventricular function subsequent to follow-up echocardiography after percutaneous coronary intervention following acute ST-elevation myocardial infarction. Exp Ther Med 2018;15:4089-96.

3. Amzulescu MS, De Craene M, Langet H, et al. Myocardial strain imaging: review of general principles, validation, and sources of discrepancies. Eur Heart J Cardiovasc Imaging 2019;20:605-19.

4. Smiseth OA, Torp H, Opdahl A, et al. Myocardial strain imaging: how useful is it in clinical decision making? Eur Heart J 2016;37:1196-207.

5. Wellnhofer E, Olariu A, Klein C, et al. Magnetic resonance low-dose dobutamine test is superior to SCAR quantification for the prediction of functional recovery. Circulation 2004;109:2172-4.

6. Bulluck H, Dharmakumar R, Arai AE, et al. Cardiovascular Magnetic Resonance in Acute ST-Segment-Elevation Myocardial Infarction: Recent Advances, Controversies, and Future Directions. Circulation 2018;137:1949-64.

7. Zou Q, Zheng T, Zhou SL, et al. Quantitative Evaluation of Myocardial Strain After Myocardial Infarction with Cardiovascular Magnetic Resonance Tissue-Tracking Imaging. Int Heart J 2020;61:429-36.

8. Kim RJ, Wu E, Rafael A, et al. The use of contrastenhanced magnetic resonance imaging to identify reversible myocardial dysfunction. $\mathrm{N}$ Engl J Med 2000;343:1445-53.

9. Muser D, Castro SA, Santangeli P, et al. Clinical applications of feature-tracking cardiac magnetic resonance imaging. World J Cardiol 2018;10:210-21.

10. Vartdal T, Brunvand H, Pettersen E, et al. Early prediction of infarct size by strain Doppler echocardiography after coronary reperfusion. J Am Coll Cardiol 2007;49:1715-21.

11. Cimino S, Canali E, Petronilli V, et al. Global and regional longitudinal strain assessed by two-dimensional speckle tracking echocardiography identifies early myocardial dysfunction and transmural extent of myocardial scar in patients with acute ST elevation myocardial infarction and relatively preserved LV function. Eur Heart J Cardiovasc Imaging 2013;14:805-11.

12. Huttin O, Marie PY, Benichou M, et al. Temporal deformation pattern in acute and late phases of STelevation myocardial infarction: incremental value of longitudinal post-systolic strain to assess myocardial viability. Clin Res Cardiol 2016;105:815-26.

13. Kramer CM, Barkhausen J, Bucciarelli-Ducci C, et al. Standardized cardiovascular magnetic resonance imaging (CMR) protocols: 2020 update. J Cardiovasc Magn Reson 2020;22:17.

14. Cerqueira MD, Weissman NJ, Dilsizian V, et al. Standardized myocardial segmentation and nomenclature for tomographic imaging of the heart. A statement for healthcare professionals from the Cardiac Imaging Committee of the Council on Clinical Cardiology of the American Heart Association. Circulation 2002;105:539-42.

15. Osman NF, Kerwin WS, McVeigh ER, et al. Cardiac motion tracking using CINE harmonic phase (HARP) magnetic resonance imaging. Magn Reson Med 1999;42:1048-60.

16. Amado LC, Gerber BL, Gupta SN, et al. Accurate and objective infarct sizing by contrast-enhanced magnetic resonance imaging in a canine myocardial infarction model. J Am Coll Cardiol 2004;44:2383-9.

17. Streiner DL. Starting at the beginning: an introduction to coefficient alpha and internal consistency. J Pers Assess 2003;80:99-103.

18. Castillo E, Osman NF, Rosen BD, et al. Quantitative assessment of regional myocardial function with MRtagging in a multi-center study: interobserver and intraobserver agreement of fast strain analysis with Harmonic Phase (HARP) MRI. J Cardiovasc Magn Reson 2005;7:783-91.

19. Schober P, Boer C, Schwarte LA. Correlation Coefficients: Appropriate Use and Interpretation. Anesth Analg 2018;126:1763-8.

20. Schuster A, Kutty S, Padiyath A, et al. Cardiovascular magnetic resonance myocardial feature tracking detects quantitative wall motion during dobutamine stress. J Cardiovasc Magn Reson 2011;13:58.

21. Eitel I, Stiermaier T, Lange T, et al. Cardiac Magnetic Resonance Myocardial Feature Tracking for Optimized Prediction of Cardiovascular Events Following Myocardial Infarction. JACC Cardiovasc Imaging 2018;11:1433-44.

22. Sjøli B, Ørn S, Grenne B, et al. Diagnostic capability and reproducibility of strain by Doppler and by speckle tracking in patients with acute myocardial infarction. JACC Cardiovasc Imaging 2009;2:24-33.

23. Li S, Zhao L, Lu A, et al. Comparison of Left Ventricular Global Strain in Anterior and Non-anterior Wall 
Myocardial Infarction With CMR Tissue Tracking. Front Physiol 2020;11:530108.

24. Mangion K, McComb C, Auger DA, et al. Magnetic Resonance Imaging of Myocardial Strain After Acute STSegment-Elevation Myocardial Infarction: A Systematic Review. Circ Cardiovasc Imaging 2017;10:e006498.

25. Riffel JH, Keller MG, Rost F, et al. Left ventricular long axis strain: a new prognosticator in non-ischemic dilated cardiomyopathy? J Cardiovasc Magn Reson 2016;18:36.

26. Hwang JW, Kim SM, Park SJ, et al. Assessment of reverse remodeling predicted by myocardial deformation on tissue tracking in patients with severe aortic stenosis: a cardiovascular magnetic resonance imaging study. J Cardiovasc Magn Reson 2017;19:80.

27. Reindl M, Tiller C, Holzknecht M, et al. Prognostic Implications of Global Longitudinal Strain by FeatureTracking Cardiac Magnetic Resonance in ST-Elevation Myocardial Infarction. Circ Cardiovasc Imaging

Cite this article as: $\mathrm{Li} \mathrm{J}, \mathrm{Li} \mathrm{M}$, Chen Y, Cheng L, Qian G, Li J, Zhou X, Liu B, Dong W. Impact of apical and mid-ventricular transmural infarcts in patients with acute myocardial infarction determined by using late gadolinium enhancement combined with feature tracking magnetic resonance. Cardiovasc Diagn Ther 2022;12(1):103-113. doi: 10.21037/cdt-21-382 2019;12:e009404.

28. Alpert JS. The pathophysiology of acute myocardial infarction. Cardiology 1989;76:85-95.

29. Ito H. No-reflow phenomenon and prognosis in patients with acute myocardial infarction. Nat Clin Pract Cardiovasc Med 2006;3:499-506.

30. Ho SY. Anatomy and myoarchitecture of the left ventricular wall in normal and in disease. Eur J Echocardiogr 2009;10:iii3-7.

31. Leitman M, Tyomkin V, Peleg E, et al. Left ventricular function in acute inflammatory peri-myocardial diseases - new insights and long-term follow-up. Cardiovasc Ultrasound 2012;10:42.

32. Dobrovie M, Barreiro-Pérez M, Curione D, et al. Intervendor reproducibility and accuracy of segmental left ventricular strain measurements using CMR feature tracking. Eur Radiol 2019;29:6846-57. 


\section{Supplementary}
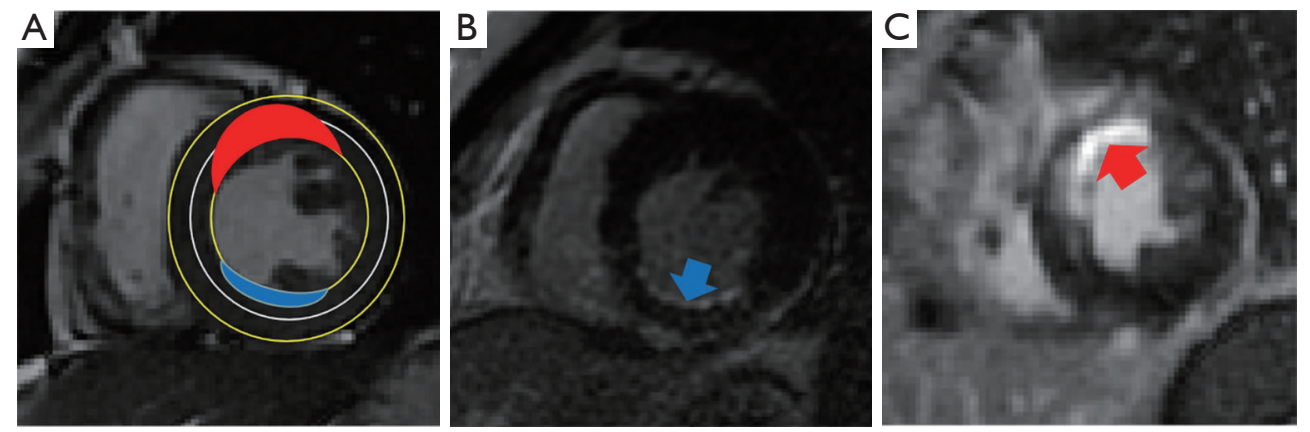

Figure S1 The segmental transmurality of myocardial infarction determined by late-gadolinium-enhanced (LGE) imaging. (A) Schematic diagram for transmurality calculations. The blue area shows the non-transmural infarction of the posterior septal and inferior wall; the red area shows the transmural infarction of the anterior septal and anterior wall. (B) LGE of the midventricular short-axis view in a patient with non-transmural infarction of the posterior septal and inferior wall (1-50\% amount of LGE). (C) LGE of the midventricular short-axis view in a patient with transmural infarction of the anterior septal and anterior wall (51-100\% amount of LGE). Arrow: LGE. 